\title{
Discussion on the Application of Rhubarb in Treatise on Febrile Diseases
}

\author{
Nan Zhao, Lanxiu Cao* \\ Shaanxi University of Chinese Medicine, Xianyang 712046, Shaanxi, China \\ *Correspondence Author
}

\begin{abstract}
By consulting ancient books and literatures, this paper probes into the law of application of Rhubarb by Zhong Jing from the aspects of compatibility and dosage of Rhubarb, and finds that the application of Rhubarb in compatibility is rigorous, especially in the application of dosage skillfully, and in the application of theory and method comprehensively, so that rhubarb where each prescription are not the same, each has its wonderful, rhubarb for the future to play an even more important role, fully used in clinical.
\end{abstract}

Keywords: Treatise on typhoid and miscellaneous diseases, Rhubarb, Compatibility, Dosage.

\section{Introduction}

Rhubarb has more than 2000 years of history, as early as in ancient times known as "Huang Liang", or say "rhubarb", "Army", "The general". Refer to ancient books, many physicians through the ages are recommended and rhubarb, all because of its function, effect is obvious. Zhang Zhongjing in Han dynasty to study writing book typhoid miscellaneous disease theory, modern physicians generally referred to as "the father of the sense". Of Zhongjing of rhubarb, especially attaches great importance to the application of rhubarb eighty-nine times in the book, there are 36 prescriptions containing rhubarb, and the use of the rule of rhubarb[1]. To grasp now, in this paper, from the aspects of compatibility, dosage of rhubarb is analyzed in this paper.

\section{Medicine for Compatibility}

2.1 Cold Approximately: The Compatibility of Rhubarb Mirabilite, Acid-insoluble Ash, Magnolia Bark

Party a large bearing gas soup, and stomach gas bearing soup, big chest soup and big chest pill. Although a total of rhubarb and mirabilite purge spilled hot performance, but rhubarb more adept at capturing scleroma, glauber's salt is good at soft firm fights. Rhubarb and mirabilite share, a hard, a soft, both crack scleroma, capturing hot junction. As Youke Wu said: “Glauber's salt...”[2]. Ke Qin once said: “Zhongjing make glauber's salt...’[3].

\subsubsection{Large and small bearing gas soup and stomach qi soup}

Large bearing gas soup and stomach gas bearing soup contains the word "bearing gas", the root in rhubarb. Large bearing gas soup and stomach gas soup has ruled out the intestinal dry excrement, drain the stomach heat treatment purposes. Large bearing gas soup, Zhongjing appointment 32 times in the book, its main function is to purge drain hot, used for intestinal dry excrement, or hot and dry hair painful inside. The party of rhubarb to xie to reduce heat knot, thanh hoa intestinal dry excrement; Glauber's salt, salty and cold, moist the knot, in night, help rhubarb xie together in addition to the intestinal lodge. Adjustable bearing gas stomach soup hot in is mainly used in stomach, intestinal tract has not fully early damage phenomenon of yang-ming hot junction. Can be used to discharge heat dryness of rhubarb and mirabilite. Little bear gas soup from the big bear gas soup remove glauber's salt in a medicine. Rhubarb in this party used as medicine, clear bowel aperient, eliminate dry excrement. Visible Zhongjing's prescription in rhubarb was particularly flexible use, how many and complex situation without medication, shall be effective as the best choice.

\subsubsection{Big chest soup, chest pill}

Rhubarb also is a very important effect on the water, in the "typhoid miscellaneous disease theory" about big chest soup with big chest pill embodied articles involved. Invaded by invagination of the pathogens of tangible form of chest syndrome, big chest soup, pill treatments are available. In accordance with the original content: "Upper thoracic belongs to Yang...[4]. Because of the difference of each person's own physique, cause disease into the cause of the differences, can be divided into two kinds, one is heat evil drink cardiac knot in the heat generated in the diaphragm. Visible "heart a little pain, touch press as hard as stone after" symptoms, priority with big chest soup eliminate pathogenic heat, water to drink. The party opened the first use of euphorbia kansui fierce power attack by drinking water, storm beat and stagnant, combining with rhubarb xie evil expelling cold, glauber's salt for purging pathogenic heat, soft firm knot. Big chest pill is bind to tonga semen lepidii, almonds, Bai Mi made into a pill. Use of rhubarb and mirabilite, euphorbia kansui is given priority to, can be spilled hot junction, dredge water hot, topped by drinking water. The compatibility of rhubarb and mirabilite, commonly used in clinical for the stagnant caused by constipation, intestinal carbuncle sore.

\subsection{Embellish Approximately: The Compatibility of Rhubarb Cannabis}

Pitted ren bolus certificate that spleen about certificate, Waitai Miyao Fang recorded: "Pockmarked benevolence bolus therapy...For the spleen about"[5]. Sayaka kernel using rhubarb pills side out features under attack, heat the folding of the fire; Cannabis ganping, can make the intestinal embellish smooth, two drug administering an embellish, defecate is unimpeded.

2.3 Crack Method: Peach Kernel Compatibility of 


\section{Rhubarb}

Rhubarb in "typhoid miscellaneous disease theory" in article 106 of the peach pit bearing gas bind, article 124 arrived when the soup with nut share all have the effect of invigorate the circulation of the blood[6]. Two parties card because the heat and blood stasis block, pulse gas bad sun fact lead to storage, so the rhubarb for here to invigorate the points and remove blood stasis and evil from hot water and can break. Have to say that Zhongjing use fine! Peach pit gas soup, cold hot water of rhubarb, medicine for king, in his own play a role for activating blood circulation and compatibility with the gentleman medicine walnuts contribute to broken blood storage work. Arrive when following the certificate is lower blood storage intensive blood stasis than evil heat, through the use of rhubarb compatibility peach kernel, shalt bind can promoting blood circulation to remove blood stasis, push the new old, directly into the blood meridian. Two prescriptions with contains, weight, medication system priorities, with the cube, is the place of Zhongjing's use.

\subsection{Temperature Approximately: The Compatibility of Radix Et Rhizoma Rhei, Radix Scutellariae, Rhizoma Coptidis Lateral Root of Aconite}

From radix et rhizoma rhei xie heart soup in radix aconiti lateralis praeparata can be seen in the use of lateral root of aconite, Zhongjing through hot rhubarb, explored the monkshood, two pills a cold one hot, go hand in hand, to play its function, Be commended especially at zaijing You: "the use of thefirst". Rhubarb soup, rhizoma coptidis xie heart substitute flooded with qing, dispel on coke, coke real hot, rhubarb xie, radix scutellariae qing, experience and the homogeneity of the two drugs, makes clear heat purging fire effect significantly.

\subsection{Clear the Method: The Compatibility of Rhubarb Gall}

Artemisia capillaris soup is mainly for damp heat jaundice disease, rhubarb soup reuse, Dan Xi Xin Fa supplementary said: "Jaundice liver taste hot and humid as for when to pass the first"[7]. By rhubarb have few points evil heat, blood stasis heat effect, thus pushing headed by rhubarb.

\section{Dose Application}

Dose particularly lofty theory of typhoid miscellaneous disease, drug use and with the change of dose produce different effect, is the so-called: "different dose, different from attending." If the drugs don't pay attention to dose, Zhongjing with party will not be realized.

\subsection{Medicinal Broth of the Dosage}

One of the main indications include blood wenjie, hot and humid indicates stagnation, phlegm retention alternating knot, muggy, blood stasis block to wait for a few kinds of inside. The typhoid miscellaneous disease theory, there are 25 one involving the use of rhubarb. Gardenia big when use rhubarb is only "one tael"; Artemisia capillaris soup etc. eight using "rhubarb two taels"; Arrive when, such as soup, four using rhubarb "three teals"; Big bearing gas soup, small, peach pit gas bearing gas soup soup, soup of adjustable gastric gas bearing 10 "rhubarb four teals"; When big chest soup, magnolia officinalis (2 songs) with "six taels". On the basis of Zhongjing with rhubarb dose on the number of parties, about between two to four taels multi-purpose.

Gardenia when big main treatment of body wet and hot, after drinking the wine of people will be hot and humid jaundice, visible "chagrin or hot pain in the heart, chest the new full or constipation." Here refer to article 154, article 155 and article 164 of the original[8], therefore syndrome is mild, the rhubarb in only one tael, can clear heat except vexed; As neijing says, reuse of rhubarb pathogens, stronger condition can affect human body again and again. so of Zhongjing medicine, take its lightweight and eliminate pathogenic and upright; such as cassia twig to increase in 279 [8] when using rhubarb, make rhubarb, into the spleen by purging the product, in addition to abdominal pain, because rhubarb not treating spleen to medicine, spleen solid abdominal pain, belongs to the lunar forward by Yang Ming, so only rhubarb two taels Daniel Yin; article 236, article 260[8] of artemisia capillaris decoction syndrome mainly hot and humid and jaundice treatment. Take two taels rhubarb Zhongjing slightly, to make it have diarrhoea damp and hot blood stasis, rather than just make defecate under attack. For xie heart bind, Synopsis of the Golden Chamber description says: "Head injury, cause vomiting blood, nosebleed, attending with xie heart soup." Due to damage blood bleeding, need immediate hemostasis, Zhongjing with "a total of three, two taels rhubarb, each a couple of rhizoma coptidis, radix scutellariae."

Large bearing gas is dry excrement and hot soup apply gas knot, illness critically ill symptom of heavy, therefore need to reuse the four taels wash gastrointestinal stagnant rhubarb, used to push the new; and real not serious, such as the early dry pathogens do not meet the real evil accumulation of small gas bearing decoction syndrome, should also be opened four taels rhubarb to reuse the heat knot, remove the tangible reality of the evil. Rhubarb soup of peony for therapy is more rapid intestinal carbuncle, acute abdominal pain so rhubarb with four taels every time; and as rhubarb saltpeter decoction syndrome is a real evil, in the vital qi is not empty, so the reuse rhubarb and table under to four taels purging effect. Postpartum disease is water with blood and blood room card need rhubarb soup of euphorbia kansui, radix et rhizoma rhei with four taels blood stasis.

Or 136 in treatise on febrile disease by big chest soup, Synopsis of the Golden Chamber records, in order to make rhubarb in these three (Yang evil invagination of chest syndrome, drink a chest full of lower abdomen and woman full like a drum sample (disease) play a spilled hot evil role, if a small amount of application, to meet the effect, so you need to use a lot. On six taels big chest soup and magnolia bark when using rhubarb.

\subsection{Dosage of Pills}

Pills in rhubarb can have the intention of slow attack, cooperate and ease his medicine, to eliminate pathogenic blood without injury is wonderful. Zhongjing Fang rhubarb pills for the agent of seven, "Three": the turtle shell decoct pill; "One tael": "Own pepper Li yellow pill"; "ten minutes": Rhubarb sting worm pill; "Three taels": Resisting pill; "Half a 
kilo": big chest pill; "A kilo": pitted ren bolus, three pre-emergency pill (didn't explain the dosage). Turtle shell decoct pill medicine, a total of 23 , long time not attending malaria rehabilitation, internal and remove lump, because just treat remove lump fever is given priority to, the junction is complementary, rhubarb attack by blood, only three points with rhubarb; Brown pill with Brown pepper in rhubarb tunnel with other fungicides, separate the intestinal moisture from the feces. With the method of small dose of frequency of attack, so the rhubarb in only one tael; Rhubarb Bolus treats seven injuries and labor disease. Syndrome differentiation and treatment of Jinguiyaolue decoction said: "The party in rhubarb swings down by stasis for you...[9]. Therefore, using rhubarb for ten minutes. Opened on pills for when water is made, the take medicine slow attack, Zhongjing with rhubarb three taels, the medicine, is unique in the broken line by blood stasis party. In the chest pill fong rhubarb xie hot junction, dosage, most actual and intention for attack.

\section{Summary}

On the theory of typhoid miscellaneous diseases, rhubarb and expounded his medicine compatibility and dose, the drug of rhubarb characteristics of different role in the party, at the same time also to other aspects of comprehensive factors. Zhongjing's use of rhubarb, wide range of uses, prescription, is unable to ratio and other drugs; and after the use of rhubarb embodies the curative effect of also bring out the best in each other. For use of rhubarb, was called the acknowledgement as example, the use of time, has joined some own ideas, to be the icing on the cake. Due to the time problem, of course, for the application of rhubarb and drawbacks, but for us to know the meaning of the rule of Zhongjing applying rhubarb have deep.

\section{References}

[1] Again Good, Zhang Guang-hao Li. The theory of plague to recreate the contribution of science of seasonal febrile diseases[J]. Journal of Traditional Chinese Medicine, 2003, 21(6): 887-889.

[2] He Yanli, Jianhua Shi, Wang Hong. The clinical application of theory of professor Jianhua Shi "moves"[J]. Misdiagnosis of China, 2018, 8.

[3] Dai Shurong. Talk about the application of rhubarb in treatise on febrile disease[J]. The Doctor of Traditional Chinese Medicine in Sichuan, 1983, 7(2): 48-49.

[4] Chen Qingxing. Treatise on febrile disease analysis rhubarb compatibility of the law $[\mathrm{J}]$. Misdiagnosis of China, 2002: 2.

[5] Wang Tao. Outside the secret s (18)[M]. China Medical Science and Technology Press, 2011: 8.

[6] Luo Xin. The application of rhubarb in treatise on febrile disease[J]. Journal of Chengdu University of Traditional Chinese Medicine, 1983, 3(4): 28-33.

[7] Yang Medicine. New on[M]. Hebei People's Publishing House, 1979:120-121.

[8] The Doctor of Traditional Chinese Medicine Research Institute. "treatise on febrile diseases", which evaluation[M]. Beijing: Chinese Press of Traditional Chinese Medicine, 2011-11.

[9] Approaches. Synopsis decoction syndrome differentiation[M]. Beijing: China Science and Technology Publishing House, 1993. 